\title{
Acute coronary syndrome featuring dynamic J waves
}

\author{
Andreas Y. Andreou ${ }^{1,2}$ \\ ${ }^{1}$ Department of Cardiology, Limassol General Hospital, Limassol, Cyprus \\ ${ }^{2}$ University of Nicosia Medical School, Nicosia, Cyprus
}

A 59-year-old male patient with a history of percutaneous coronary intervention (PCI) to the left anterior descending (LAD) artery presented because of angina at rest. Admission electrocardiography (ECG) (Fig. 1A) revealed at least $0.05 \mathrm{mV}$ ST-segment depression at the J point in leads II, aVF, aVL, I, and V3-V6, at least $0.1 \mathrm{mV}$ ST-segment elevation (STE) at the J point in leads aVR and V1 with STE in lead aVR > V1 and notch-type J waves in leads aVL and I. Coronary angiography performed due to refractory angina showed highgrade lesions in the LAD and left circumflex (LCx) arteries. Electrocardiography (Fig. 1B) after PCI to a proximal LCx artery culprit lesion (Fig. 1C) revealed resolution of ST-segment changes, reduction of $\mathrm{J}$ waves amplitude in lead aVL and disappearance of $\mathrm{J}$ waves in lead I. His serum troponin I peaked at $3.5 \mathrm{ng} / \mathrm{mL}$ (normal $<0.5 \mathrm{ng} / \mathrm{mL}$ ). He was discharged following staged PCI of the LAD artery lesions (Fig. 1C).

Ischemia-induced $\mathrm{J}$ waves have been widely reported in vasospastic angina and less frequently in acute myocardial infarction; in both these clinical entities, they have been shown to confer an increased risk of ventricular fibrillation. They are registered in ECG leads facing the ischemic territory and followed by STE in persistent ischemia. Accordingly, ischemia-induced $\mathrm{J}$ waves would be useful in localizing the infarct-related artery. The dynamic J waves presented herein were clearly ischemia-induced as no other conditions reported to induce J waves such as hypothermia, hypercalcemia or Brugada syndrome were documented. This case highlights that dynamic $\mathrm{J}$ waves registered in leads aVL and I in patients with acute coronary syndrome suggest the presence of a culprit lesion in the LCx artery.

Conflict of interest: None declared

Address for correspondence: Andreas Y. Andreou, MD, FESC, FACC, FSCAI, Department of Cardiology, Limassol General Hospital, Nikeas street, Pano Polemidia, Postal code 3304, PO Box 56060, Limassol, Cyprus, tel: +357-25801437, fax: +357-25801432, e-mail: y.andreas@yahoo.com

Received: 22.11.2020 Accepted: 6.02.2021

This article is available in open access under Creative Common Attribution-Non-Commercial-No Derivatives 4.0 International (CC BY-NC-ND 4.0) license, allowing to download articles and share them with others as long as they credit the authors and the publisher, but without permission to change them in any way or use them commercially. 

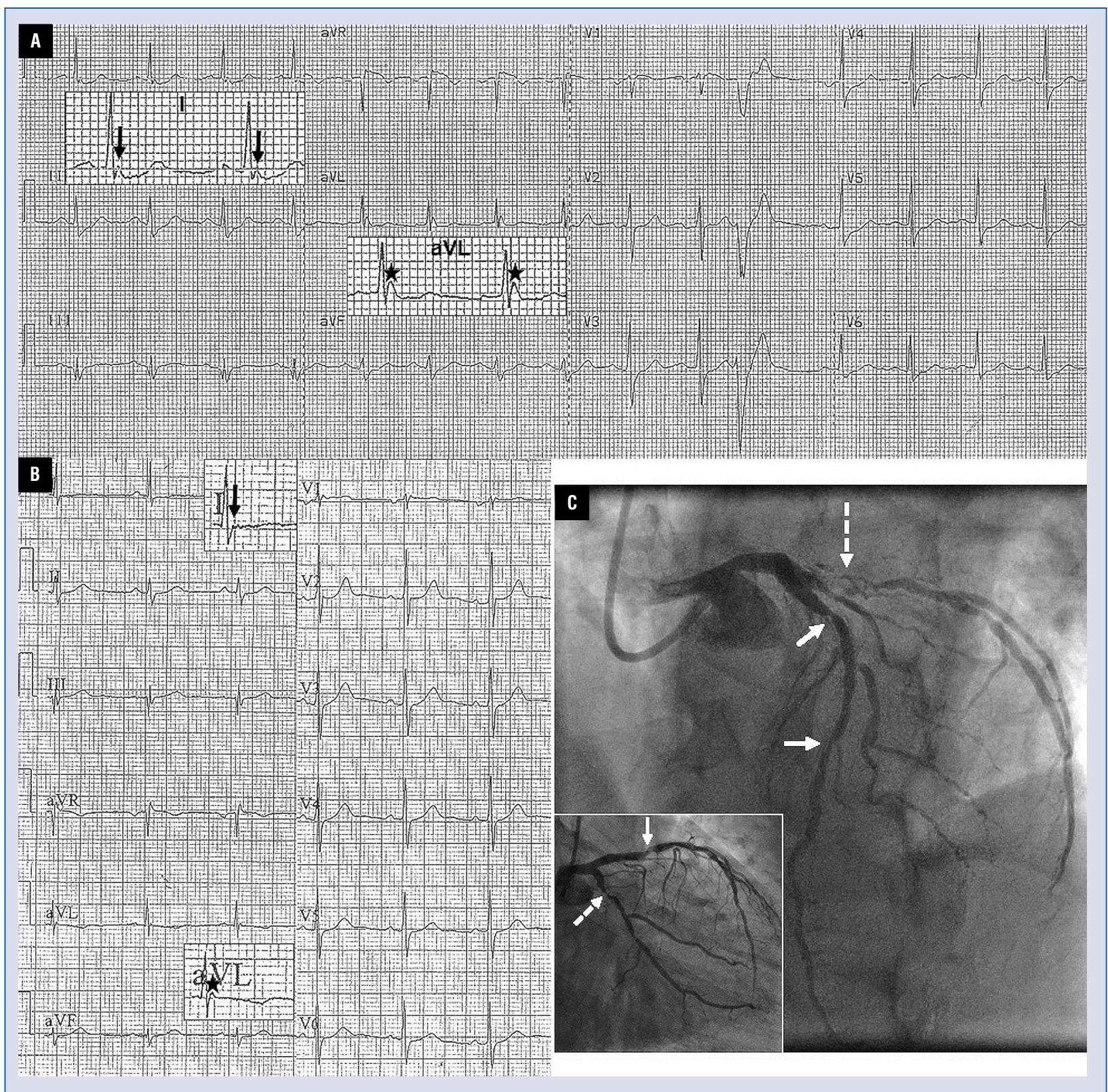

Figure 1. Electrocardiogram (ECG) on admission (A) and after percutaneous coronary intervention (PCI) to the culprit artery (B) and coronary angiographic images at baseline (C). ECG on admission: note the notch-type J waves in lead I (embedded panel: arrows) and in lead aVL (embedded panel; asterisks) where they were particularly prominent $(>0.1 \mathrm{mV}$ ). ECG after PCI to a left circumflex (LCX) artery culprit lesion depicted $\mathrm{J}$ waves of reduced amplitude $(<0.1 \mathrm{mV}$ ) in lead aVL (embedded panel; asterisk) and absent $\mathrm{J}$ waves in lead I (embedded panel; arrow). Note, that leads V2 and V3 have been reversed. Baseline coronary angiography depicted a high-grade, hazy stenosis with irregular borders (dotted arrows) in the proximal LCx artery (culprit lesion) and bystander high-grade lesions in the left anterior descending artery (solid arrows). 